Микола Борисович Нікулін (канд. техн. наук, доцент) Ігор Володимирович Ромашко

Полтавський національний технічний університет імені Юрія Кондратюка, Полтава, Украӥна

\title{
ОЦІНКА ЗА ПІКФАКТОРОМ ШИРОКОСМУГОВИХ СИГНАЛІВ В БАЗИСІ ФУНКЦІЙ ЛЯГГЕРА
}

У ряді робіт, присвячених питанням синтезу і обробки складних сигналів, разом з тригонометричним базисом використовуються і інші ортогональні базиси. Проте, в даних роботах, часто, не дається оцінка таких сигналів за пікфактором, а також має місие застосування ортогональних базисів не на кінцевому інтервалі, що приводить до різного роду труднощів під час рішення задач формування $i$ обробки сигналів.

Метою даної статті є розгляд питання синтезу складних сигналів в ортогональних базисах Ляггера для класу складних сигналів. Зазначений синтез широкосмугових сигналів представляє інтерес для систем радіозв'язку.

Ключові слова: базис функиій Лягера, пікфактор, сигнал, модулячія.

\section{Вступ}

Сучасні системи радіозв'язку використовують широкосмугові сигнали враховуючи їх значні переваги на вузькосмуговими, що визначає необхідність синтезу зазначених сигналів.

Постановка проблеми.

Якщо при проведенні синтезу складних сигналів разом 3 тригонометричним базисом використовується і інші базиси, необхідно давати оцінку таких сигналів, враховуючи пікфактор та застосовуючи ортогональний базис на кінцевому інтервалі.

Аналіз остатніх досліджень і публікацій

Останнім часом для синтезу широкосмугових сигналів використовувався тригонометричний базис, де апаратна платформа використовувала класичну елементну базу. Синтез смугових сигналів в базисі функцій Лягера забезпечує побудову пристроїв модуляції на основі RCелементів, що значно спрощує апаратну реалізацію модуляторів.

Виклад основного матеріалу дослідження.

Якщо в якості несущого коливання як і раніше використовувати відрізок гармоніки, а огинаючу формувати в ортонормованом на інтервалі $[0, \mathrm{~T}]$ базисі, відмінному від тригонометричного, тодіпри балансній модуляції гармонійну несучу функцію визначаємо наступним співвідношенням

$$
\mathrm{E}_{\mathrm{r}}(\mathrm{t})=\sum_{\mathrm{k}=\mathrm{k}_{\mathrm{r} 1}}^{\mathrm{k}_{\mathrm{r} 2}} \mathrm{~A}_{\mathrm{rk}} \varphi_{\mathrm{rk}}(\mathrm{t}), 0 \leq \mathrm{t} \leq \mathrm{T},
$$

r - ий варіант передаваємого складного сигналу представляється у формі :

$$
\begin{aligned}
\mathrm{U}_{\mathrm{r}}(\mathrm{t})= & \sum_{\mathrm{k}=\mathrm{k}_{\mathrm{r} 1}}^{\mathrm{k}_{\mathrm{r} 2}} \mathrm{~A}_{\mathrm{rk}} \varphi_{\mathrm{rk}}(\mathrm{t}) \cos \left(\omega_{\mathrm{H}} \mathrm{t}+\varphi_{0}\right), 0 \leq \mathrm{t} \leq \mathrm{T}, \text { (2) } \\
& \text { де }\left\{\varphi_{\mathrm{rk}}(\mathrm{t})\right\}-\text { координатні функції, }
\end{aligned}
$$

належні ортонорморваному на інтервалі $[0, \mathrm{~T}]$ базису, відмінному від тригонометричного;
$A_{r k}$ - амплітуда к- тої координатної функції; $\varphi_{0}$ - початкова фаза високочастотного заповнення сигналу.

У разі синтезу ортогональних сигналів (2.2), координатні функції $\mathrm{r}$ i $\ell$ варіантів повинні задовольняти умові.

$$
\int_{0}^{T} \varphi_{r k}(t) \varphi_{1 k}(t) \mathrm{dt}=0 \quad \text { при } \quad \mathrm{r} \neq \ell(3)
$$

Розглянемо формування широкосмугових сигналів в базисі функцій Ляггера 3 точки зору ортогональності і ортнормованості.

Практичний інтерес до функцій Ляггера під час рішення задач синтезу i обробки сигналів обумовлений тим, що вони можуть бути реалізовані як імпульсні реакції порівняно простих фізичних ланцюгів кінцевого порядку.ФункціїЛяггера визначаються рівнянням:

$$
\ell_{k}(t)=\frac{1}{k !} \ell^{-t / 2} L_{k}(t)
$$

де $L_{k}-$ к- ий поліном Ляггера отриманий в результаті рішення лінійного диференціального рівняння другого порядку:

$$
x y+(1-t) y^{\prime}+k y=0
$$

і можуть бути визначені на підставі наступного виразу:

$$
\mathrm{L}_{\mathrm{k}}(\mathrm{t})=(-1)^{\mathrm{k}} \ell^{\mathrm{t}} \frac{\mathrm{d}^{(\mathrm{k})}}{\mathrm{dt}^{\mathrm{k}}}\left(\mathrm{t}^{\mathrm{k}} \ell^{-\mathrm{t}}\right)
$$

Під час рішення задач синтезу ортогональних фільтрів широко використовуються функції Ляггера виду:

$\varphi_{\mathrm{k}}(\mathrm{t})=\frac{\ell^{-\beta \mathrm{t}}}{\mathrm{k} !} \mathrm{L}_{\mathrm{k}}(2 \beta \mathrm{t})(6)$

що допускають за рахунок речовинного позитивного параметра $\beta$ зручне тимчасове масштабування. 
3 врахуванням (2.6) вираз для $\varphi_{k}(t)$ перетвориться до наступної форми:

$$
\begin{aligned}
& \varphi_{\mathrm{k}}(\mathrm{t})=\sqrt{2 \beta} \ell^{-\beta \mathrm{t}} \\
& \frac{(2 \beta)^{\mathrm{k}}}{\mathrm{k} !} \mathrm{t}^{\mathrm{k}}-\frac{\mathrm{k}(2 \beta)^{\mathrm{k}-1}}{(\mathrm{k}-1) !} \mathrm{t}^{\mathrm{k}-1}+\frac{\mathrm{k}(\mathrm{k}-1)(2 \beta)^{\mathrm{k}-2}}{2 !(\mathrm{k}-2) !} \mathrm{t}^{\mathrm{k}-2}- \\
& -\frac{\mathrm{k}(\mathrm{k}-1)(\mathrm{k}-2)(2 \beta)^{\mathrm{k}-3}}{3 !(\mathrm{k}-3) !} \mathrm{t}^{\mathrm{k}-3} \ldots .(-1)^{\mathrm{k}}
\end{aligned}
$$

Дані функції $\left\{\varphi_{\mathrm{k}}(\mathrm{t})\right\}$ утворюють ортнормовану систему на інтервалі $t \in[0, \infty]$, тобто

$\int_{0}^{\infty} \varphi_{\mathrm{k}}(\mathrm{t}) \varphi_{\mathrm{n}}(\mathrm{t}) \mathrm{dt}=\left\{\begin{array}{l}1_{-} \text {при }{ }_{-} \mathrm{k}=\mathrm{n} \\ 0_{-} \text {при _ } \mathrm{k} \neq \mathrm{n}\end{array}\right.$

Декілька перших функцій Ляггера згідно (7) рівні:

$$
\begin{aligned}
& \varphi_{1}(\mathrm{t})=\sqrt{2 \beta} \ell^{-\beta \mathrm{t}}(2 \beta \mathrm{t}-1), \\
& \varphi_{2}(\mathrm{t})=\sqrt{2 \beta} \ell^{-\beta \mathrm{t}}\left(2 \beta^{2} \mathrm{t}^{2}-4 \beta \mathrm{t}+1\right), \\
& \varphi_{3}(\mathrm{t})=\sqrt{2 \beta} \ell^{-\beta \mathrm{t}}\left(\frac{4}{3} \beta^{3} \mathrm{t}^{3}-6 \beta^{2} \mathrm{t}^{2}+6 \beta \mathrm{t}-1\right) .
\end{aligned}
$$

Розглянемо синтез складних сигналів паралельної структури в базисі функції Ляггера.

Відповідно до (1) модулююча функція, використана для формування r-говаріанту сигналу, визначається співвідношенням:

$$
\mathrm{E}_{\mathrm{r}}(\mathrm{t})=\sum_{\mathrm{k}=\mathrm{k}_{\mathrm{r} 1}}^{\mathrm{k}_{\mathrm{r} 2}} \mathrm{~A}_{\mathrm{rk}} \varphi_{\mathrm{rk}}(\mathrm{t}), 0 \leq \mathrm{t} \leq \mathrm{T}(9)
$$

Під час безпосереднього підставлення функції Ляггера (7) в (9), унаслідок неузгодженості їх інтервалу ортогональності 3 кінцевою тривалістю сигналу виникають шуми неортогональності:

$$
\int_{0}^{\mathrm{T}} \varphi_{\mathrm{k}}(\mathrm{t}) \varphi_{\mathrm{n}}(\mathrm{t}) \mathrm{dt} \neq 0 \text { при } \mathrm{n} \neq \mathrm{k}
$$

Також необхідно відзначити, що і норма $\varphi_{\mathrm{k}}(\mathrm{t})$ на кінцевому інтервалі є відмінною від 1.

$$
\int_{0}^{\mathrm{T}} \varphi_{\mathrm{k}}(\mathrm{t}) \varphi_{\mathrm{n}}(\mathrm{t}) \mathrm{dt} \neq 1 \quad \text { при } n=k
$$

внаслідок чого виникають помилки неортонормальності.

Отже, під час синтезу сигналів в базисі функції Ляггера необхідна мінімізація шумів не ортогональності і помилок неортонормальності.

Проаналізуємо залежність шумів неортогональності, визначених відповідно до (10) і шумів неортонормальності рівних

$$
\mathrm{C}_{\mathrm{kk}}=1-\left|\int_{0}^{\mathrm{T}} \varphi_{\mathrm{k}}^{2}(\mathrm{t}) \mathrm{dt}\right|(12)
$$

від параметра $\beta$.

Таким чином, вибором величини параметра $\beta$ рівні даних шумів i помилок можуть бути приведені до допустимих значень.

Відповідно неортогональності демодулятора спільно 3 помилками неортонормальності представляють перехідну перешкоду вигляду:

$$
\begin{gathered}
\mathrm{Q}_{\mathrm{n}}=\sum_{\mathrm{i}, \mathrm{j}=\mathrm{k}_{\mathrm{r} 1}}^{\mathrm{k}_{\mathrm{r} 2}} \int_{0}^{\mathrm{T}} \varphi_{\mathrm{i}}(\mathrm{t}) \varphi_{\mathrm{j}}(\mathrm{t}) \mathrm{dt}+\sum_{\mathrm{i}, \mathrm{j}=\mathrm{k}_{\ell 1}}^{\mathrm{k}_{\ell 2}} \int_{0}^{\mathrm{T}} \varphi_{\mathrm{i}}(\mathrm{t}) \varphi_{\mathrm{j}}(\mathrm{t}) \mathrm{dt}+ \\
+\sum_{\mathrm{k}=\mathrm{k}_{\mathrm{r} 1}}^{\mathrm{k}_{\mathrm{r} 2}}\left\{1-\left|\int_{0}^{\mathrm{T}} \varphi_{\mathrm{kr}}^{2}(\mathrm{t}) \mathrm{dt}\right|\right\}+\sum_{\mathrm{k}=\mathrm{k}_{\ell 1}}^{\mathrm{k}_{\ell 2}}\left\{1-\left|\int_{0}^{\mathrm{T}} \varphi_{\mathrm{k} \ell}(\mathrm{t}) \mathrm{dt}\right|\right\}+ \\
+2 \sum_{\mathrm{k}=\mathrm{k}_{\mathrm{r} 1}}^{\mathrm{k}_{\mathrm{r} 2}} \sum_{\mathrm{k}=\mathrm{k}_{\ell 1}}^{\mathrm{k}_{\ell 2}} \int_{0}^{\mathrm{T}} \varphi_{\mathrm{kr}}(\mathrm{t}) \varphi_{\mathrm{k} \ell}(\mathrm{t}) \mathrm{dt} .(13)
\end{gathered}
$$

При виконанні умови:

$\mathrm{Q}_{\mathrm{n}}^{2} \leq 0.01\left(\mathrm{~K}_{\mathrm{r} 2}-\mathrm{K}_{\mathrm{r} 1}+1\right)^{2}$

для вірогідності помилки $\mathrm{P} \geq 10^{-6}$, порушеннями неортогональності інеортонормальності можна знехтувати.

Отже, на підставі (14), може бути визначений допустимий рівень перехідної перешкоди $\mathrm{Q}_{n . д о n}$, а з обліком (13) при відомих координатних функціях $\left\{\varphi_{\mathrm{k}}(\mathrm{t})\right\}_{\mathrm{k}_{\mathrm{r} 1}}^{\mathrm{k}_{\mathrm{r} 2}}$, використовуваних для формування r, - го і $\ell$ - го варіантів сигналу i необхідна величина параметра $\beta$. При цьому шуми неортогональності $C_{n \kappa}$ для $n+\kappa \leq 10$ можуть бути розраховані за наступною формулою: $C_{n k}=l^{-2 \beta T}\{$

$$
\begin{aligned}
& -\frac{2^{\mathrm{k}+\mathrm{n}}}{\mathrm{k} ! \mathrm{n} !}(\beta \mathrm{T})^{\mathrm{n}+\mathrm{k}}+\frac{\mathrm{n}(\mathrm{n}-1)+\mathrm{k}(\mathrm{k}-1)}{\mathrm{k} ! \mathrm{n} !}(2 \beta \mathrm{T})^{\mathrm{n}+\mathrm{k}-1}+\{ \\
& \frac{k^{2}(k-1)^{2}(k-2)^{2}+n^{2}(n-1)^{2}(n-2)^{2}+3 n^{2} k^{2}\left[(k-1)^{2}+(n-1)^{2}\right]}{6 \mathrm{k} ! n !}+
\end{aligned}
$$$$
+\frac{\left.\left.6 k^{2}+n^{2}\right)(k+n-1)(n+k-2)-6 n+k\right)(n+k-1)(n+k-2)}{6 ! n !}-
$$$$
-\frac{3\left[n^{2}(n-1)^{2}+k^{2}(k-1)^{2}+2 n^{2} k^{2}\right](k+n-2)}{6 k ! n !}
$$

\}$\left.(2 \beta \mathrm{T})^{\mathrm{n}+\mathrm{k}-3}\right\}$

отриманою в результаті обчислення (10).

Даний вираз при $\mathrm{n}=$ к може бути використано i для обчислення помилок неортонормальності $\mathrm{C}_{\mathrm{nn}}$.

Відомо, що під час формування складних сигналів велике значення має забезпечення на інтервалі тривалості сигналу $[0, \mathrm{~T}]$ рівномірно мінімального пікфактора. Це диктується необхідністю раціонального використання потужності передавача і запасу лінійності тракту приймача.

Вочевидь, для складних сигналів паралельної структури (2) рівномірно мінімальний пікфактор може бути забезпечений відповідним вибором форми моделюючої функції $\mathrm{E}_{\mathrm{r}}(\mathrm{t})$. Також необхідно відзначити, що разом 3 балансною 
модуляцією може здійснюватися i амплітудна модуляція несучої. Отже, необхідно здійснити не тільки вибір форми $\mathrm{E}_{\mathrm{r}}(\mathrm{t})$, але i найбільш переважний, з погляду забезпечення мінімального пікфактора, від модуляції.

Форма $\mathrm{E}_{\mathrm{r}}(\mathrm{t})$ залежить від бази, рівної

$\mathrm{B}=\mathrm{k}_{\mathrm{r} 2}-\mathrm{k}_{\mathrm{r} 1}+1$ i конкретних координатних

функцій, використаних під час формування. На рис 1 побудовані графіки функцій Ляггера

другого і третього порядку.

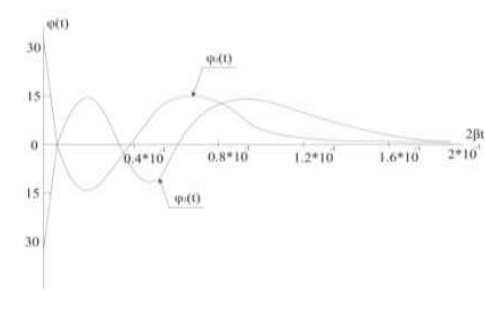

Функиія Лягтера

Рис 1

3 графіків видно, що дані функції мають екстремуми при $\mathrm{t}=0$. Причому, величина екстремуму для парної функції складає $+\sqrt{2 \beta}$, а для непарної функції складає $-\sqrt{2 \beta}$. На підставі цього, неважко побачити, що якщо під час формування $\mathrm{E}_{\mathrm{r}}(\mathrm{t})$ число функції Ляггера парного порядку не буде рівне числу функції непарного порядку, то екстремальне значення $\mathrm{E}_{\mathrm{r}}(\mathrm{t})$ також складатиме величину, рівну $\pm \sqrt{2 \beta}$. За невиконанням даної умови абсолютна величина екстремального значення модулюючої функції складає величину менше $|\sqrt{2 \beta}|$.

Отже, для забезпечення мінімального пікфактора сигналів (2.2) необхідно забезпечити рівність числа парних і непарних функцій Ляггера, використовуваних під час формування $\mathrm{E}_{\mathrm{r}}(\mathrm{t})$.

Пікфактор сигналів при амплітудній i балансній модуляціях, відповідно, рівний

$$
\begin{aligned}
& \prod_{\mathrm{AM}}=\frac{\left.\left[\mathrm{U}_{\mathrm{mH}}+\max _{\mathrm{t} \in[0, \mathrm{~T}]} \mid \mathrm{E}_{\mathrm{r}}(\mathrm{t})\right]\right] \sqrt{2}}{\sqrt{\mathrm{U}_{\mathrm{mH}}^{2}+\gamma+\frac{\mathrm{B}}{\mathrm{T}}}} \\
& \prod_{\text {БM }}=\frac{\max \left|\mathrm{E}_{\mathrm{r}}(\mathrm{t})\right| \sqrt{2}}{\sqrt{\frac{\mathrm{B}}{\mathrm{T}}}}
\end{aligned}
$$

\section{Jimepamypa}

1. Семенов А. М., Сикарев А. А. Широкополосная радиосвязь //Радиосвязь, -1985г. ,278с.. 2. Сикарев А. А., Лебедев О.Н. Микроелектронные устройства формирования и обработки сложных сигналов де $\mathrm{U}_{\mathrm{mH}}$ - амплітуда несучої $\gamma=\frac{\mathrm{U}_{\mathrm{mH}}}{\mathrm{T}} \int_{0}^{\mathrm{T}} \mathrm{E}_{\mathrm{r}}(\mathrm{t}) \mathrm{dt}$.

Результати розрахунку, відповідно до (16) i (17), при $\beta=500$ і a=10, приведені в таблиці 1., де $\Pi_{\mathrm{E}_{\mathrm{r}(\mathrm{t})}}$ - пікфактор $\mathrm{E}_{\mathrm{r}}(\mathrm{t})$.

Таблиця 1 - Результати розрахунку пікфактора амплітудної і балансної модуляції

\begin{tabular}{|c|c|c|c|}
\hline $\mathrm{E}_{\mathrm{r}}(\mathrm{t})$ & & & \\
& & & \\
\hline$\varphi_{2}(\mathrm{t})+\varphi_{3}(\mathrm{t})$ & .4 & .46 & .74 \\
& 4 & & \\
\hline$\varphi_{2}(\mathrm{t})+\varphi_{3}(\mathrm{t})$ & .3 & .65 & .58 \\
$+\varphi_{4}(t)$ & 5 & & \\
\hline$\varphi_{2}(\mathrm{t})+\varphi_{3}(\mathrm{t})+\varphi_{4}(\mathrm{t})$ & .1 & .08 & .47 \\
$+\varphi_{5}(\mathrm{t})$ & 9 & & \\
\hline$\varphi_{1}(\mathrm{t})+\varphi_{2}(\mathrm{t})+\varphi_{3}(\mathrm{t})+\varphi_{4}(\mathrm{t})+\varphi_{5}(\mathrm{t})+\varphi_{6}(\mathrm{t})$ & .9 & .72 & .216 \\
& 2 & & \\
\hline
\end{tabular}

Аналіз набутих значень ще раз підтверджує необхідність рівності числа функцій Ляггера парного i непарного порядку при формуванні $\mathrm{E}_{\mathrm{r}}(\mathrm{t})$, а також указує, що при малих значеннях $\Pi_{\mathrm{E}_{\mathrm{r}(\mathrm{t})}}$ пікфактора моделюючої функції

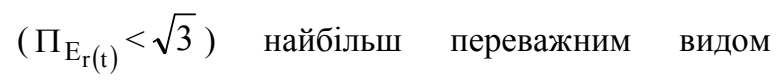
модуляції є балансна.

Таким чином, за умови відповідного вибору параметра $\beta$, на основі функцій Ляггера може бути здійснений синтез складних сигналів паралельної структури із задовільним пікфактором.

\section{Висновки й перспективи подальших}

\section{досліджень}

Отже, для забезпечення мінімального пікфактора необхідно забезпечити рівність числа парних і непарних функцій Ляггера. При малих значеннях пікфактора моделюючої функції найбільш переважним видом модуляції є балансна. Таким чином, за умови відповідного вибору параметра $\beta$, на основі функцій Ляггера може бути здійснений синтез складних сигналів паралельної структури із задовільним пікфактором.

\section{ОЦЕНКА ПО ПИКФАКТОРУ ШИРОКОПОЛОСОВИХ СИГНАЛОВ В БАЗИСЕ ФУНКИЙ ЛЯГГЕРА}


Николай Борисович Никулин (канд. техн. наук, доцент)

Ігорь Владимирович Ромашко

Полтавский национальный технический университет имени Юрия Кондратюка, Полтава, Украина

В последнее время в ряде работ, посвященных вопросам синтеза и обработки сложных сигналов, вместе с тригонометрическим базисом используются и другие ортогональные базисы. Однако, в данных работах, часто, не дается оценка таких сигналов по пикфактору, а также имеет место применение ортогональных базисов не в конечном интервале, что приводит кразличного рода трудностям при решении задач формирования и обработки сигналов.

Целью данной статьи является рассмотрение вопроса синтеза сложных сигналов в ортогональных базисах Лягzера для класса сложных сигналов. Указанный синтез широкополосных сигналов представляет интерес для систем радиосвязи.

Ключевые слова: базис функиий Лягера, пикфактор, сигнал, модуляциия

\section{ESTIMATION BY THE PICK FACTOR OF BROADBAND SIGNALS IN THE BASIS OF THE FUNNY LEGGER \\ Nikolai B. Nikulin (candidate of technical sciences, associate professor) Igor V. Romashko}

\section{Poltava National Technical University named after Yuri Kondratyuk, Poltava, Ukraine}

Recently, in a number of works devoted to the synthesis and processing of complex signals, along with the trigonometric basis, other orthogonal bases are also used. However, in these works, often, such signals are not evaluated by the peak factor, and also the use of orthogonal bases does not occur in the finite interval, which leads to a variety of difficulties in solving the problems of signal generation and processing.

The purpose of this article is to consider the synthesis of complex signals in orthogonal Laguerre bases for a class of complex signals. This synthesis of broadband signals is of interest for radio communication systems..

Keywords: basis of Laguerre functions, peakfactor, signal, modulation.

\section{References}

1.Semenov AM, Sikarev AA Broadband radio communication // Radiocommunication, -1985. ,Pp. 278. 2. Sikarev AA, Lebedev ON Microelectronic devices for the formation and processing of complex signals // Radiocommunication, $\quad 1988, \quad$ Pp. 239
3. Dovgy S.O. Telecommunications of Ukraine // Tehnika, -2001., Pp. 533. 4. Yanke EM, Emde FS, Lesch F.V. Specialfunctions//Science,-1964, Pp.343 